\title{
Kernos
}

Revue internationale et pluridisciplinaire de religion grecque antique

6 | 1993

Varia

\section{Calame, Thésée et l'imaginaire athénien. Légende et culte en Grèce antique}

\section{Vinciane Pirenne-Delforge}

\section{(2) OpenEdition \\ Journals}

Édition électronique

URL : http://journals.openedition.org/kernos/567

DOI : 10.4000/kernos.567

ISSN : 2034-7871

Éditeur

Centre international d'étude de la religion grecque antique

Édition imprimée

Date de publication : 1 janvier 1993

Pagination : 385-388

ISSN : 0776-3824

\section{Référence électronique}

Vinciane Pirenne-Delforge, «C. Calame, Thésée et l'imaginaire athénien. Légende et culte en Grèce antique », Kernos [En ligne], 6 | 1993, mis en ligne le 07 avril 2011, consulté le 24 septembre 2020. URL : http://journals.openedition.org/kernos/567 ; DOI : https://doi.org/10.4000/kernos.567 
Claude CALAME, Thésée et l'imaginaire athénien. Légende et culte en Grèce antique (Préface de Pierre Vidal-Naquet), Lausanne, Éditions Payot, 1990, 1 vol. 14 x 22,5 cm, 480 p. (Coll. Sciences humaines). ISBN : 2-601-03080-1.

Comme le souligne Pierre Vidal-Naquet dans la préface, Claude Calame étudie ici un mythe héroïque inscrit dans l'imaginaire politique et la pratique rituelle. Mais l'enjeu est bien plus large que la compréhension de la figure de Thésée et de ses relations avec les Athéniens : ce sont une fois de plus les catégories du mythe et du rite qui sont mises en question dans cette enquête. Par bonheur, les réflexions théoriques, souvent difficiles, du premier chapitre prennent vie tout au long des cinq autres parties qui tentent d'apporter des réponses aux questions suivantes: 1) pourquoi avoir fait d'un récit légendaire l'aition d'une série d'actes cultuels dans des circonstances historiques précises ? 2) quand et pourquoi est intervenue cette opération particulière d'insertion d'une pratique dans un discours légendaire ? 3) quelle est la spécificité de chacun de ces ordres de la manifestation culturelle et qu'est-ce qui a permis leur mise en perspective mutuelle?

Plutôt que de fixer l'objectif sur une définition du mythe et du rite, et après avoir passé en revue quelques grands devanciers autour de cette problématique, l'A. s'attache, dans le 1er chapitre intitulé "Créations symboliques", au fonctionnement général de ces catégories : mythe et rite sont deux manifestations parmi d'autres de la pensée symbolique et leur mise en relation dépend de chaque culture - autrement dit, rien de nécessaire entre eux; un tel processus d'élaboration intellectuelle s'effectue par l'énonciation de propositions - globalement arbitraires dont la fonction première est de construire un ordre qui intègre les événements disparates et incohérents produits au sein du monde naturel ou social. Dès lors, la différence entre rite et mythe tient au mode d'expression employé : "expression somatique recourant à des entités concrètes d'un côté, expression à travers la langue de l'autre" (p. 54). Cependant, dans le cas de la Grèce ancienne, les rites nous sont connus au travers de récits et leur appréhension passe dès lors aussi par le langage. Ce premier chapitre se referme sur une présentation de l'approche sémiotique proposée qui, pour un lecteur novice en ces matières, ne trouvera vraiment son sens que dans l'application qui en est faite à la légende de Thésée.

L'analyse syntaxique de la légende et des rites se répartit sur les chapitre II et III, intitulés respectivement "Les aventures de Thésée» et "Les cultes "fondés" par Thésée». En premier lieu, la légende est présentée dans toutes ses variantes - neuf versions de l'étape insulaire qui voit Ariane abandonnée! -, avec comme récit de référence la Vie de Thésée de Plutarque. Ensuite, on passe à la description narrative des 
cultes, assez décevante, comme le souligne l'A. lui-même. Les rites n'ayant pas de logique narrative autonome, ils sont insérés dans un récit qui doit donner un sens à leur caractère périodique en projetant dans le passé l'acte fondateur. L'A., appliquant rigoureusement sa méthode, émet l'hypothèse que Plutarque aurait pu inventer des rites et, inversement, s'étonne de l'absence de lien opéré entre certain rite connu et un épisode de la légende. L'application des principes théoriques initiaux permet ainsi l'énoncé d'hypothèses intéressantes et la grande rigueur de Claude Calame empêche cette application de devenir caricaturale et conjecturale. Ces deux chapitres sont donc essentiellement descriptifs et il faut attendre les quatrième et cinquième parties pour entamer ce que l'A. appelle "les conséquences d'ordre sémantique» de la mise en relation du récit et des actions.

Le chapitre IV intitulé "Figures mythiques d'un héros national" scrute chaque épisode de la biographie de Thésée pour mettre en évidence l'évolution du héros, tant d'un point de vue biologique que civique et politique. L'intervention de certains dieux, en certains temps et lieux n'est pas indifférente. Que ce soit Apollon ou Aphrodite, Zeus ou Poséidon, Dionysos ou Athéna, que ce soit aux marges de l'Attique, lors du voyage de Trézène à Athènes, ou en mer et sur les îles, lors de la traversée vers le labyrinthe crétois et l'affrontement du Minotaure, chaque intervention trouve une juste place pour converger enfin vers Athènes et l'accession à la royauté, au pouvoir. Le deuxième pan de la biographie du héros, qui comprend des actes aussi peu glorieux que l'enlèvement d'Hélène enfant par un Thésée vieillissant ou l'aide à Pirithoos pour enlever Perséphone, constitue une véritable descente aux Enfers - au propre comme au figuré... - qui se termine par une mort peu glorieuse en terre marginale. C'est donc essentiellement sur le cœur de la légende que se concentrera l'héroïsation et le culte.

Le chapitre $\mathrm{V}$ tente quant à lui de pallier la maigreur de l'analyse syntaxique des cultes. Intitulé "Cycle et espace cultuels athéniens", il propose une remarquable analyse des fêtes du calendrier athénien en relation - directe ou indirecte - avec la saga de Thésée. Ainsi Pyanopsies et Thargélies, Oschophories et Anthestéries, Skirophories ou Skira, Cybernésia, Thesmophories, Halôa se trouvent engagées, à des titres divers et en des lieux divers en fonction des dieux sollicités, dans la célébration de l'agriculture, de l'alimentation civilisée et du commerce maritime. L'étude attentive de la géographie festive conduit à la mise en place de six cercles concentriques dont le cour est une Acropole irradiant vers ce qui devient peu à peu, dans le temps de l'histoire, l'«empire» athénien.

Et le mot est lâché : l'histoire. C'est à l'ancrage historique de la légende qu'est consacré le sixième et dernier chapitre intitulé "L'héroïsation symbolique dans l'histoire». L'A. s'attache à repérer les 
circonstances d'ordre politique et social dont légende et cultes portent la trace et dans lesquelles ils s'inscrivent. S'érigeant contre la mise en exergue exclusive de personnages éminents (Thémistocle, Cimon, Périclès...) ou d'événements singuliers (Guerres médiques, Guerre du Péloponnèse...) pour expliquer la promotion du personnage et la «focalisation» attique de sa légende, Claude Calame, puisant dans les définitions préalables, conçoit l'élaboration symbolique comme le produit des moments de crise politique connus par le système démocratique athénien : “C'est dans la perspective de ce décalage idéalisant qu'on tentera de comprendre comment, à partir non seulement de l'univers d'expérience de la communauté, mais en particulier d'éléments légendaires ou cultuels préexistants, la pensée symbolique a pu être sollicitée par le développement progressif de la puissance athénienne pour élargir et resémantiser mythe et rite, légende et culte» (p. 420). On assiste donc, pour faire bref, à la mise en perspective du parcours terrestre de Thésée avec la délimitation du territoire de l'Attique, et à celle de son parcours maritime avec l'expansion d'Athènes en mer Égée, processus auquel il faut ajouter l'Amazonomachie et la Centauromachie en regard des Guerres médiques et des prétentions athéniennes sur le Bosphore. «La narration de la légende et la pratique cultuelle [toutes deux préexistantes] convergent dans la manipulation de la réalité socio-culturelle» (p. 444).

Le livre est dense, complexe en ses préalables méthodologiques et remarquablement documenté. Les notes - malheureusement rejetées à la fin de chaque chapitre - abondent en précisions et développements complémentaires, et tant les sources anciennes que les références aux travaux modernes ${ }^{16}$ forment une assise très sûre à un discours où aucune question - et elles sont nombreuses - n'est escamotée. S'inscrivant dans la problématique difficile des rapports entre le mythe, le rite et l'histoire, ce travail est d'une très grande cohérence, même si l'on peut parfois regretter que le découpage - obligé ? - en analyse syntaxique/analyse sémantique entraîne de nombreuses répétitions. On soulignera encore la qualité de l'analyse des fêtes athéniennes qui fait litière d'un certain nombre d'idées reçues comme, par exemple, le caractère initiatique des Oschophories. Tant pour l'étude de l'idéologie athénienne aux époques archaíque et classique que pour la compréhension de ce Thésée multiforme, le livre de Claude Calame est désormais un passage obligé. Un regret cependant : l'absence d'index, même si les subdivisions abondent dans le texte; à moins que ce choix ne soit

16 Une absence cependant, p. 277, n. 106. G. Dontas (The True Aglaurion, in Hesperia, 52 [1983], p. 48-63) a reposé la question de la localisation de l'ancienne agora qui détermine la topographie du centre politique de l'Athènes archaique. 
délibéré pour inviter le lecteur à prendre connaissance de l'ensemble de la démarche ${ }^{17}$.

\section{Vinciane PIRENNE-DELFORGE (Liège)}

Figures grecques de l'intermédiaire, sous la direction de Claude CALAME, Études de Lettres, Université de Lausanne, 1992, 146 p.

Claude Calame a rassemblé, dans un numéro de la revue Études de Lettres, cinq articles aux thèmes très différents mais à la démarche comparable, et dont la plupart, nous prévient l'éditeur, sont issus de travaux de mémoires de licence.

Il s'agit, dans chaque étude, de dépasser le concept binaire du «même» et de l'«autre» pour démontrer, explicitement ou implicitement, que la pensée des Grecs ne fonctionnait pas selon un mode aussi simple, aussi tranché, et que le modèle structuraliste, trop réducteur, n'est pas toujours approprié pour en rendre compte : «si la pensée polaire souvent à l'œuvre en Grèce a pu faire croire que les oppositions mises à jour par l'analyse structurale traditionnelle bénéficiaient d'un fondement structurel, il est temps de constater que les Grecs se gardaient bien de penser le monde selon les canons de la logique binaire" (Cl. CALAME).

Partant, l'intérêt du recueil est double : dans la plupart des cas il offre, sur le sujet traité, de nouveaux éclairages; en outre, son apport sur le plan de la réflexion méthodologique est indéniable.

Dans un article intitulé Figures du tyran archaïque : entre le monstre et le sage, Christine YERLY analyse la représentation du tyran dans la poésie archaïque. Le tyran, tantôt monstreux, tantôt modèle de sagesse, est relégué aux marges de la société, de la normalité, mais son rôle d'arbitre et la transition qu'il assure entre pouvoir absolu et démocratie le réintègrent dans la cité.

Pierre VOELKE, dans Ambivalence, médiation, intégration : à propos de l'espace dans le drame satyrique, montre, par l'étude des données mêmes des textes conservés, que l'espace géographique où se déroule l'action des pièces n'est pas seulement l'emblème du monde sauvage opposé au monde civilisé, mais qu'il représente un lieu de passage de l'un à l'autre.

17 Quelques erreurs et coquilles au passage : p. 16 : dans lesquelles et non dans laquelle; p. $37, \S 3.3$. : mise au jour et non mise à jour; p. $42,2 \mathrm{e} \S:$ mettre au jour et non mettre à jour; p. 103, § 5.3. : pouvoir et non pouvir; p. 144, $\$ 1.2 .1$. : on va parler et non on vas parler; p. 152, $\S 1.4 .2$. : Souda et non Suda; p. 322, 1er $\S$ : civilisatrice et non cvilisatrice; p. 388, n. 85 : Pithoïgia et non Phitoïgia. 